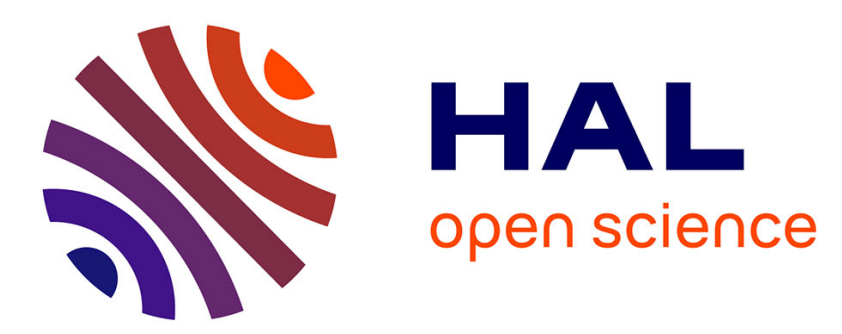

\title{
Stabilization of nonlinear systems subject to actuator saturation
}

\author{
Souad Bezzaoucha, Benoît Marx, Didier Maquin, José Ragot
}

\section{To cite this version:}

Souad Bezzaoucha, Benoît Marx, Didier Maquin, José Ragot. Stabilization of nonlinear systems subject to actuator saturation. International Conference on Fuzzy System, FUZZ 2013, Jul 2013, Hyderabad, India. pp.889-894. hal-00812955

\section{HAL Id: hal-00812955 https://hal.science/hal-00812955}

Submitted on 26 Nov 2021

HAL is a multi-disciplinary open access archive for the deposit and dissemination of scientific research documents, whether they are published or not. The documents may come from teaching and research institutions in France or abroad, or from public or private research centers.
L'archive ouverte pluridisciplinaire HAL, est destinée au dépôt et à la diffusion de documents scientifiques de niveau recherche, publiés ou non, émanant des établissements d'enseignement et de recherche français ou étrangers, des laboratoires publics ou privés. 


\title{
Stabilization of Nonlinear Systems Subject to Actuator Saturation
}

\author{
Souad Bezzaoucha*†, Benoît Marx* ${ }^{* \dagger}$, Didier Maquin*†, José Ragot*† \\ *Université de Lorraine, CRAN, UMR 7039, 2 avenue de la Forêt de Haye, Vandoeuvre-lès-Nancy Cedex, 54516, France \\ ${ }^{\dagger} \mathrm{CNRS}, \mathrm{CRAN}, \mathrm{UMR}$ 7039, France. \\ e-mail:firstname.name@univ-lorraine.fr.
}

\begin{abstract}
This paper addresses the stabilization of nonlinear systems described by Takagi-Sugeno models affected by input actuator saturation. A parallel distributed compensation design is used for the state feedback controller. Stabilization conditions in the sense of the Lyapunov method are derived and expressed as a linear matrix inequality problem. The obtained gains depend on the actuator saturation limits. A cart-pendulum example is presented to illustrate the effectiveness of the proposed approach.
\end{abstract}

Keywords-Takagi-Sugeno systems, actuator saturation, parallel distributed compensation control law

\section{INTRODUCTION}

Actuator saturation or control input saturation is probably one of the most usual nonlinearity encountered in control engineering due to the physical impossibility of applying unlimited control signals and/or safety constraints.

In general, there are two main design strategies to deal with actuator saturations. The first strategy is a two-step approach in which a nominal linear controller is first constructed by ignoring the actuator saturation. Then, after this controller has been designed, usually using standard linear design tools, a so called anti-windup compensator is designed to handle the saturation constraints [1], [2], [3], [4]. A typical anti-windup scheme consists in augmenting a nominal pre-designed linear controller with a compensator based on the discrepancy between unsaturated and saturated control signals fed to the plant [5]. The second strategy considers the saturation constraint from the beginning of the design task. Several approaches were developed, one of them is the invariant sets framework, which has been significantly developed in control engineering over the last decades, see [6], [7]. This framework ensures that every state trajectory that starts inside the invariant set will not exceed it, i.e. the system states will be bounded inside this set.

An interesting approach for the determination of the invariant sets framework was applied in [8] and [9]. This approach consists in the so-called polytopic rewriting of the saturation constraint. This polytopic representation is then used to determinate (maximize) the largest invariant set in which the system states remain bounded.

In the contrary of the previous method, that most likely ensures to never reach the saturation level in order to preserve the closed loop performances, in this paper, we explicitly consider the saturation constraint, and even admit its occurrence. In fact, the authors use the Takagi-Sugeno (T-S) representation of the saturation (as well known as the polytopic representation) to integrate the limitation constraints into the control synthesis, such that the system stability is ensured and the control gains are calculated depending on the saturation level.

The authors would like to precise that even if the expressions of the T-S saturation has some similarity to the polytopic one used in [8] and [9], the development, control strategy and objectives are completely different since in the proposed approach the invariant sets are never considered; moreover the objective is the stabilization of nonlinear systems represented with the Takagi-Sugeno (T-S) models and the synthesis of a state feedback controller by parallel distributed compensation (PDC) with control gains explicitly depending on the saturation level. The controller is subject to actuator saturation and the input saturation is directly taken into account in the controller design. Stabilization conditions are derived from the Lyapunov method and expressed as linear matrix inequalities (LMI). A cart-pendulum example is considered to illustrate the effectiveness of the proposed approach. The nonlinear equations of the cart-pendulum are given and, using the Sector Nonlinearity Transformation (SNT) [10], [11], a T-S model of the system is deduced and used. The system is subject to actuator saturations, it will be shown that these input constraints may cause the instability of the nonlinear system, but with the proposed approach the stability of the closed-loop system is ensured.

The rest of this paper is organized as follows. Section 2 introduces the Takagi-Sugeno structure for modeling and some preliminary results, mathematical notations and a brief description of the saturation. It is followed by the representation of the nonlinear saturation by a T-S structure in section 3 . In section 4 is designed a state feedback control law depending on the saturation bounds. A numerical example and some simulation results are given in section 5. Conclusions and future works are exposed in section 6 .

\section{PREliminaries}

\section{A. Takagi-Sugeno structure for modeling}

Initially introduced in [12], the T-S models represent a simple and accurate method to study the nonlinear behaviors. The T-S modeling allows to represent the behavior of nonlinear systems by the interpolation of a set of linear submodels [10], [13], [14]. Each submodel contributes to the global behavior of the nonlinear system through a weighting function $\mu_{i}(\xi(t))$. The T-S structure is given by 


$$
\left\{\begin{array}{l}
\dot{x}(t)=\sum_{i=1}^{n} \mu_{i}(\xi(t))\left(A_{i} x(t)+B_{i} u(t)\right) \\
y(t)=\sum_{i=1}^{n} \mu_{i}(\xi(t))\left(C_{i} x(t)+D_{i} u(t)\right)
\end{array}\right.
$$

where $x(t) \in \mathbb{R}^{n_{x}}$ is the system state variable, $u(t) \in \mathbb{R}^{n_{u}}$ is the control input and $y(t) \in \mathbb{R}^{m}$ is the system output. $\xi(t) \in$ $\mathbb{R}^{q}$ is the decision variable vector assumed to be measurable (as the system output) or known (as the system input). The weighting functions $\mu_{i}(\xi(t))$ of the $n$ submodels satisfy the convex sum property

$$
\left\{\begin{array}{l}
\sum_{i=1}^{n} \mu_{i}(\xi(t))=1 \\
0 \leq \mu_{i}(\xi(t)) \leq 1, \quad i=1, \ldots, n
\end{array}\right.
$$

In the remaining of the paper, the following lemmas are used:

Lemma 1. Consider two matrices $X$ and $Y$ with appropriate dimensions and $G$ a symmetric positive definite matrix. The following property is verified

$$
X^{T} Y+Y^{T} X \leq X^{T} G X+Y^{T} G^{-1} Y
$$

Lemma 2. (Congruence) Consider two matrices $X$ and $Y$, if $X$ is positive (resp. negative) definite and if $Y$ is a full column rank matrix, then the matrix $Y X Y^{T}$ is positive (resp. negative) definite.

\section{B. Mathematical notations}

The following notations are used throughout the paper: a block diagonal matrix with the square matrices $A_{1}, \ldots, A_{n}$ on its diagonal is denoted $\operatorname{diag}\left(A_{1}, \ldots, A_{n}\right)$. For any matrix, $M, \mathbb{S}(M)$ is defined by $\mathbb{S}(M)=M+M^{T}$. The smallest and largest eigenvalues of the matrix $M$ are respectively denoted $\lambda_{\min }(M)$ and $\lambda_{\max }(M)$. The saturation function for a signal $\nu(t)$ is defined by (4), where $\nu_{\max }$ and $\nu_{\min }$ denote the saturation levels.

$$
\operatorname{sat}(\nu(t)):=\left\{\begin{array}{lll}
\nu(t) & \text { if } \quad \nu_{\min } \leq \nu(t) \leq \nu_{\max } \\
\nu_{\max } & \text { if } \quad \nu(t)>\nu_{\max } \\
\nu_{\min } & \text { if } \quad \nu(t)<\nu_{\min }
\end{array}\right.
$$

\section{PROBLEM STATEMENT}

\section{A. Takagi-Sugeno saturation control}

The main idea of this work is to model the nonlinear actuator saturation using the Takagi-Sugeno representation (section II-A) and then propose a PDC control law ensuring stability of the closed loop system. For that, it is proposed to re-write the saturation equation (4) for each component of the control input vector under a particular form.

Let us consider a control input vector $u(t) \in \mathbb{R}^{n_{u}}$, defined by

$$
u(t)=\left(\begin{array}{lll}
u_{1}(t) & \ldots & u_{n_{u}}(t)
\end{array}\right)^{T}
$$

The control input under actuator saturation constraint is given by

$$
u_{\text {sat }}(t)=\left(\begin{array}{lll}
u_{\text {sat }}^{1}(t) & \ldots & u_{\text {sat }}^{n_{u}}(t)
\end{array}\right)^{T}
$$

where $u_{\text {sat }}^{j}=\operatorname{sat}\left(u_{j}(t)\right)$, for $j=1, \ldots, n_{u}$.

Considering the three parts of the saturated signal (4), each component of the vector $u_{\text {sat }}(t)$ is written as

$$
u_{\text {sat }}^{j}(t)=\sum_{i=1}^{3} \mu_{i}^{j}\left(u_{j}(t)\right)\left(\lambda_{i}^{j} u_{j}(t)+\gamma_{i}^{j}\right), \quad j=1, \ldots, n_{u}
$$

with

$$
\begin{array}{ccc}
\lambda_{1}^{j}=0 & \lambda_{2}^{j}=1 & \lambda_{3}^{j}=0 \\
\gamma_{1}^{j}=u_{\min }^{j} & \gamma_{2}^{j}=0 & \gamma_{3}^{j}=u_{\max }^{j}
\end{array}
$$

and the weighting functions defined by

$$
\left\{\begin{array}{l}
\mu_{1}\left(u_{j}(t)\right)=\frac{1-\operatorname{sign}\left(u_{j}(t)-u_{\min }^{j}\right)}{2} \\
\mu_{2}\left(u_{j}(t)\right)=\frac{\operatorname{sign}\left(u_{j}(t)-u_{\min }^{j}\right)-\operatorname{sign}\left(u_{j}(t)-u_{\max }^{j}\right)}{2} \\
\mu_{3}\left(u_{j}(t)\right)=\frac{1+\operatorname{sign}\left(u_{j}(t)-u_{\max }^{j}\right)}{2}
\end{array}\right.
$$

Based on the convex sum property of the weighting functions (2), the control input vector $u(t) \in \mathbb{R}^{n_{u}}$ subject to actuator saturation can be written in order to have the same activation functions for all the input vector components as:

$$
\left(\begin{array}{c}
\left(\sum_{i=1}^{3} \mu_{i}^{1}\left(\lambda_{i}^{1} u_{1}(t)+\gamma_{i}^{1}\right)\right) \times\left(\prod_{k=2}(t)=\right. \\
\vdots \\
\left.\left(\sum_{i=1}^{3} \mu_{i}^{\ell}\left(\lambda_{i}^{\ell} u_{\ell}(t)+\gamma_{i}^{\ell}\right)\right) \times\left(\prod_{k=1, k}^{k} \sum_{k}(t)\right)\right) \\
\vdots \\
\left(\sum_{i=1}^{3} \mu_{j}^{n_{u}} \mu_{j}^{n_{u}}\left(\lambda_{i}^{n_{u}} u_{k}(t)\right)\right. \\
\left.\left.n_{n_{u}}(t)+\gamma_{i}^{n_{u}}\right)\right) \times\left(\prod_{k=1}^{n_{u}-1} \sum_{j=1}^{3} \mu_{j}^{k} u_{k}(t)\right)
\end{array}\right)
$$

For $n_{u}$ inputs, $3^{n_{u}}$ submodels are obtained. Thus, it is important to note that (11) is an analytical expression of the actuators saturation directly expressed in term of the control variable, that can also be expressed as

$$
u_{\text {sat }}(t)=\sum_{i=1}^{3^{n_{u}}} \mu_{i}(t)\left(\Lambda_{i} u(t)+\Gamma_{i}\right)
$$

The global weighting functions $\mu_{i}(t)$, the matrices $\Lambda_{i} \in$ $\mathbb{R}^{n_{u} \times n_{u}}$ and vectors $\Gamma_{i} \in \mathbb{R}^{n_{u} \times 1}$ are defined as follows

$$
\left\{\begin{aligned}
\mu_{i}(t) & =\prod_{j=1}^{n_{u}} \mu_{\sigma_{i}^{j}}^{j}\left(u_{j}(t)\right) \\
\Lambda_{i} & =\operatorname{diag}\left(\lambda_{\sigma_{i}^{j}}^{j}\right) \\
\Gamma_{i} & =\left[\gamma_{\sigma_{i}^{1}}^{1}, \ldots, \gamma_{\sigma_{i}^{n} u}^{n_{u}}\right]^{T}
\end{aligned}\right.
$$

where the indexes $\sigma_{i}^{j}\left(i=1, \ldots, 3^{n_{u}}\right.$ and $\left.j=1, \ldots, n_{u}\right)$, equal to 1,2 or 3 , indicate which partition of the $j$ th input $\left(\mu_{1}^{j}, \mu_{2}^{j}\right.$ or $\left.\mu_{3}^{j}\right)$ is involved in the $i^{\text {th }}$ submodel.

The relations between $i$ the number of the submodel) and the $\sigma_{i}^{j}$ indices are given by the following equation 


$$
i=3^{n_{u}-1} \sigma_{i}^{1}+3^{n_{u}-2} \sigma_{i}^{2}+\ldots+3^{0} \sigma_{i}^{n_{u}}-\left(3^{1}+3^{2}+\ldots+3^{n_{u}-1}\right)
$$

The indices $\sigma_{i}^{j}$ are such that $\left(\left(\sigma_{i}^{1}-1\right), \ldots,\left(\sigma_{i}^{n_{u}}-1\right)\right)$ correspond to (i-1) in base 3 .

An illustrative example is given for two inputs $\left(n_{u}=2\right)$, with

$$
u_{\text {sat }}(t)=\left(\begin{array}{ll}
u_{\text {sat }}^{1}(t) & u_{\text {sat }}^{2}(t)
\end{array}\right)^{T}
$$

Since three partitions are defined for each input, the TakagiSugeno model for $u_{\text {sat }}(t)$ is then represented by $3^{2}$ submodels

$$
u_{s a t}(t)=\sum_{i=1}^{9} \mu_{i}(t)\left(\Lambda_{i} u(t)+\Gamma_{i}\right)
$$

with the parameters $\mu_{i}, \Lambda_{i}$ and $\Gamma_{i}$ given by the following table

\begin{tabular}{|c|c|c|c|c|}
\hline submodel i & $\left(\sigma_{i}^{1}, \sigma_{i}^{2}\right)$ & $\mu_{i}(t)$ & $\Lambda_{i}$ & $\Gamma_{i}$ \\
\hline 1 & $(1,1)$ & $\mu_{1}^{1} \mu_{1}^{2}$ & $\operatorname{diag}\left(\lambda_{1}^{1}, \lambda_{1}^{2}\right)$ & {$\left[\begin{array}{cc}\gamma_{1}^{1} & \gamma_{1}^{2}\end{array}\right]^{T}$} \\
\hline 2 & $(1,2)$ & $\mu_{1}^{1} \mu_{2}^{2}$ & $\operatorname{diag}\left(\lambda_{1}^{1}, \lambda_{2}^{2}\right)$ & {$\left[\begin{array}{ll}\gamma_{1}^{1} & \gamma_{2}^{2}\end{array}\right]^{T}$} \\
\hline 3 & $(1,3)$ & $\mu_{1}^{1} \mu_{3}^{2}$ & $\operatorname{diag}\left(\lambda_{1}^{1}, \lambda_{3}^{2}\right)$ & {$\left[\begin{array}{ll}\gamma_{1}^{1} & \gamma_{3}^{2}\end{array}\right]^{T}$} \\
\hline 4 & $(2,1)$ & $\mu_{2}^{1} \mu_{1}^{2}$ & $\operatorname{diag}\left(\lambda_{2}^{1}, \lambda_{1}^{2}\right)$ & {$\left[\begin{array}{cc}\gamma_{2}^{1} & \gamma_{1}^{2}\end{array}\right]^{T}$} \\
\hline 5 & $(2,2)$ & $\mu_{2}^{1} \mu_{2}^{2}$ & $\operatorname{diag}\left(\lambda_{2}^{1}, \lambda_{2}^{2}\right)$ & {$\left[\begin{array}{cc}\gamma_{2}^{1} & \gamma_{2}^{2}\end{array}\right]^{T}$} \\
\hline 6 & $(2,3)$ & $\mu_{2}^{1} \mu_{3}^{2}$ & $\operatorname{diag}\left(\lambda_{2}^{1}, \lambda_{3}^{2}\right)$ & {$\left[\begin{array}{ll}\gamma_{2}^{1} & \gamma_{3}^{2}\end{array}\right]^{T}$} \\
\hline 7 & $(3,1)$ & $\mu_{3}^{1} \mu_{1}^{2}$ & $\operatorname{diag}\left(\lambda_{3}^{1}, \lambda_{1}^{2}\right)$ & {$\left[\begin{array}{ll}\gamma_{3}^{1} & \gamma_{1}^{2}\end{array}\right]^{T}$} \\
\hline 8 & $(3,2)$ & $\mu_{3}^{1} \mu_{2}^{2}$ & $\operatorname{diag}\left(\lambda_{3}^{1}, \lambda_{2}^{2}\right)$ & {$\left[\begin{array}{ll}\gamma_{3}^{1} & \gamma_{2}^{2}\end{array}\right]^{T}$} \\
\hline 9 & $(3,3)$ & $\mu_{3}^{1} \mu_{3}^{2}$ & $\operatorname{diag}\left(\lambda_{3}^{1}, \lambda_{3}^{2}\right)$ & {$\left[\begin{array}{ll}\gamma_{3}^{1} & \gamma_{3}^{3}\end{array}\right]^{T}$} \\
\hline
\end{tabular}

TABLE I. WeIghting FUnCtIONS, MATRICES $\Lambda_{i}$ AND $\Gamma_{i}$, FOR $n_{u}=2$

\section{B. Problem statement}

Let us now consider a T-S nonlinear system represented by the following state equation

$$
\dot{x}(t)=\sum_{i=1}^{n} \mu_{i}(\xi(t))\left(A_{i} x(t)+B_{i} u(t)\right)
$$

The control input $u(t)$ is subject to actuator saturation, then the system (16) becomes

$$
\dot{x}(t)=\sum_{i=1}^{n} \mu_{i}(\xi(t))\left(A_{i} x(t)+B_{i} u_{s a t}(t)\right)
$$

From (12), equation (17) can be written as

$$
\dot{x}(t)=\sum_{i=1}^{n} \sum_{k=1}^{3^{n_{u}}} \mu_{i}(\xi(t)) \mu_{k}^{s a t}(t)\left(A_{i} x(t)+B_{i}\left(\Lambda_{k} u(t)+\Gamma_{k}\right)\right)
$$

with $n$ the number of sub-models, $n_{u}$ the dimension of the input vectors, $\mu_{i}(\xi)$ and $\xi$ respectively are the system weighting functions and the measurable decision variables. $\mu_{k}^{s a t}, \Lambda_{k}$ and $\Gamma_{k}$ are respectively the saturation weighting functions and the parameters defined by equations (13).

\section{SATURATED STATE FEEDBACK CONTROL INPUT}

The objective is to design a stabilizing time-varying state feedback controller ensuring the stability of the system, even in the presence of control input saturation. The solution is obtained by representing the saturation as a T-S system and by solving an optimization problem under LMI constraints.

In the nominal case, if no saturation is affecting the system (1), the well-known following PDC (Parallel distributed compensation) can be applied

$$
u(t)=-\sum_{j=1}^{n} \mu_{j}(\xi(t)) K_{j} x(t)
$$

Using the quadrative Lyapunov function

$$
V(x(t))=x^{T}(t) P x(t), \quad P=P^{T}>0
$$

the stability of the closed-loop system is obtained by computing the gains $K_{j}$ from [10]

$P_{1} A_{i}^{T}+A_{i} P_{1}-R_{j}^{T} B_{i}^{T}-B_{i} R_{j}<0 \quad i=1, \ldots, n ; j=1, \ldots, n$

with $K_{j}=P_{1}^{-1} R_{j}$ (where $P_{1}=P^{-1}$ ).

In the presence of input saturation, the gains computed from (21) do not ensure the closed-loop stability. The objective is then to design a nonlinear state feedback controller (19) in order to guarantee the stability of the saturated system (18) such that the control gains $K_{i}$ depend on the saturation limits. By replacing the control law (19) in the T-S system (18), the obtained closed-loop system is the following

$$
\begin{aligned}
\dot{x}(t)=\sum_{i=1}^{n} \sum_{j=1}^{n} \sum_{k=1}^{3^{n u}} \mu_{i}(\xi(t)) \mu_{j}(\xi(t)) \mu_{k}^{s a t}(t) \\
\left(\left(A_{i}-B_{i} \Lambda_{k} K_{j}\right) x(t)+B_{i} \Gamma_{k}\right)
\end{aligned}
$$

The determination of the controller gains is now detailed.

Theorem 1. There exists a time-varying state feedback controller (19) for a saturated input system (18) ensuring that the system state converges toward an origin-centred ball of radius bounded by $\beta$ if there exists $P_{1}=P_{1}^{T}>0, R, \Sigma_{k}=\Sigma_{k}^{T}>0$ solutions of the following optimization problem

$$
\min _{P_{1}, R, \Sigma_{k}} \beta
$$

s.t.

$$
\left(\begin{array}{cc}
Q_{i j k} & I \\
I & -\beta I
\end{array}\right)<0
$$

with

$$
Q_{i j k}=\left(\begin{array}{cc}
\mathbb{S}\left(A_{i} P_{1}-B_{i} \Lambda_{k} R_{j}\right) & I \\
I & -\Sigma_{k}
\end{array}\right)
$$

and

$$
\Gamma_{k}^{T} B_{i}^{T} \Sigma_{k} B_{i} \Gamma_{k}<\beta
$$

for $i=1, \ldots, n, j=1, \ldots, n, k=1, \ldots, 3^{n_{u}}$.

The controller gains are given by

$$
K_{j}=P_{1}^{-1} R_{j}
$$

Proof: 
Let us define the quadratic Lyapunov function (20). According to (22) and (20), the time derivative of $V(x(t))$ is given by

$$
\begin{gathered}
\dot{V}(x(t))=\sum_{i=1}^{n} \sum_{j=1}^{n} \sum_{k=1}^{3^{n_{u}}} \mu_{i}(\xi(t)) \mu_{j}(\xi(t)) \mu_{k}^{s a t}(t) \\
\left(\mathbb{S}\left(x^{T}(t) P B_{i} \Gamma_{k}+x^{T}(t) P\left(A_{i}-B_{i} \Lambda_{k} K_{j}\right) x(t)\right)\right)
\end{gathered}
$$

Using Lemma 1, it follows that

$$
\mathbb{S}\left(x^{T}(t) P B_{i} \Gamma_{k}\right) \leq \Gamma_{k}^{T} B_{i}^{T} \Sigma_{k} B_{i} \Gamma_{k}+x^{T}(t) P \Sigma_{k}^{-1} P x(t)
$$

The time derivative of the Lyapunov function (28) is bounded as follows

$$
\begin{gathered}
\dot{V}(x(t)) \leq \sum_{i=1}^{n} \sum_{j=1}^{n} \sum_{k=1}^{3^{n_{u}}} \mu_{i}(\xi(t)) \mu_{j}(\xi(t)) \mu_{k}^{s a t}(t) \\
\left(x^{T}(t)\left(\mathbb{S}\left(P\left(A_{i}-B_{i} \Lambda_{k} K_{j}\right)+P \Sigma_{k}^{-1} P\right) x(t)+\Gamma_{k}^{T} B_{i}^{T} \Sigma_{k} B_{i} \Gamma_{k}\right)\right.
\end{gathered}
$$

Let us define

$$
\begin{gathered}
\mathcal{Q}_{i j k}=\mathbb{S}\left(P\left(A_{i}-B_{i} \Lambda_{k} K_{j}\right)\right)+P \Sigma_{k}^{-1} P \\
\varepsilon=\min _{i=1: n, j=1: n, k=1: 3^{n_{u}}} \lambda_{\min }\left(-\mathcal{Q}_{i j k}\right) \\
\delta=\max _{i=1: n, k=1: 3^{n_{u}}} \Gamma_{k}^{T} B_{i}^{T} \Sigma_{k} B_{i} \Gamma_{k}
\end{gathered}
$$

Since $\Sigma_{k}>0$ and from equation (30), according to Lyapunov stability theory [15], $\dot{V}(t)<-\varepsilon\|x\|^{2}+\delta$. It follows that $\dot{V}(t)<0$ for

$$
\left\{\begin{array}{c}
\mathcal{Q}_{i j k}<0 \\
\text { and } \\
\|x\|^{2}>\frac{\delta}{\varepsilon}
\end{array}\right.
$$

which means that $x(t)$ is uniformly bounded and converges to a small origin-centered ball of radius $\sqrt{\frac{\delta}{\varepsilon}}$.

Applying Lemma $2, \mathcal{Q}_{i j k}<0$ is equivalent to

$$
\mathbb{S}\left(P^{-1} A_{i}^{T}-P^{-1} K_{j}^{T} \Lambda_{k}^{T} B_{i}^{T}\right)+\Sigma_{k}^{-1}<0
$$

Using the variable changes

$$
\left\{\begin{array}{l}
P_{1}=P^{-1} \\
R_{j}=K_{j} P_{1}
\end{array}\right.
$$

condition (35) is linearized as

$$
\begin{aligned}
\mathbb{S}\left(P_{1} A_{i}^{T}-R_{j}^{T} \Lambda_{k}^{T} B_{i}^{T}\right)+\Sigma_{k}^{-1}<0 \\
\quad i=1, \ldots, n ; j=1, \ldots, n ; k=1, \ldots, 3^{n_{u}}
\end{aligned}
$$

Applying Schur's complement to (37), it becomes

$$
Q_{i j k}=\left(\begin{array}{cc}
\mathbb{S}\left(P_{1} A_{i}^{T}-R_{j}^{T} \Lambda_{k}^{T} B_{i}^{T}\right) & I \\
I & -\Sigma_{k}
\end{array}\right)<0
$$

As the weighting functions satisfy (2) and $\Sigma_{k}>0$, if (38) is satisfied for $i=1, \ldots, n, j=1, \ldots, n, k=1, \ldots, 3^{n_{u}}$, and $\|x\|^{2}>\frac{\delta}{\varepsilon}$, then $\dot{V}(x(t))<0$, which implies that $x(t)$ converges to an origin centered ball of radius $\sqrt{\frac{\delta}{\varepsilon}}$.

The objective is now to minimize the radius $\sqrt{\frac{\delta}{\varepsilon}}$. Firstly $\delta$ is bounded by $\beta$ (33) and the LMIs (26). From (24), with a Schur complement, it obviously follows that

$$
(1 / \beta) I<-Q_{i j k}, i=1, \ldots, 3^{n_{u}}
$$

implying that all the eigenvalues of $\left(-Q_{i j k}\right)$ are larger that $1 / \beta$. As a consequence $1 / \beta<\varepsilon$ holds, and finally the radius is bounded by $\beta$.

Remark 1. It is important to highlight that the proposed approach ensures the stability of nonlinear systems that may be destabilized by saturated control with a control proportional to the saturation limits. If the submodels are initially unstable, the proposed approach is not suitable since the LMI condition cannot be fulfilled. Thus, to overcome the submodels stability constraint, a solution would be to consider a dynamic output feedback control instead of the state feedback one [16].

\section{NumericAl EXAMPLE}

Let us consider a nonlinear cart-pendulum system illustrated in figure 1 .

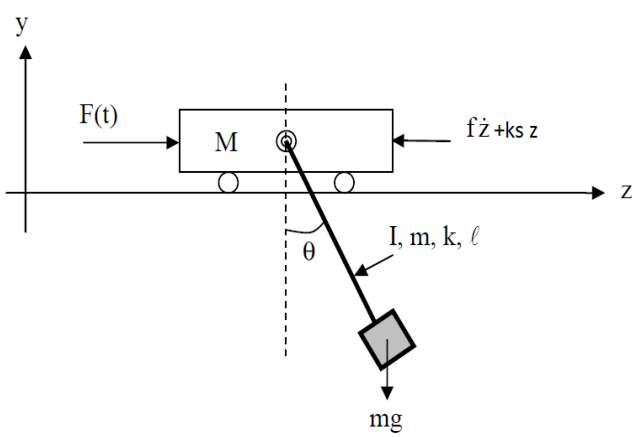

Fig. 1. Cart-pendulum system

The pendulum rotates in a vertical plan around an axis located on a cart. The cart can move along a horizontal rail, lying in the rotation plane. The state of the system is a vector $x=\left(\begin{array}{llll}x_{1} & x_{2} & x_{3} & x_{4}\end{array}\right)^{T}$ where $x_{1}=z(t)$ is the cart position, $x_{2}=\dot{z}(t)$ the cart velocity, $x_{3}=\theta(t)$ is the angle between the downward direction and the pendulum $\left(x_{2}=0\right.$ for the upright position of the pendulum), and $x_{4}=\dot{\theta}(t)$ is the pendulum angular velocity. A control force $F(t)$ parallel to the rail is applied to the cart. The pendulum mass and cart mass are denoted $m=1 K g$ and $M=5 K g$ respectively. $l=0.1 m$ is the pendulum length and $I$ the moment of inertia of the pendulum with respect to its axis on the cart. The cart friction is composed of the viscous friction proportional to the cart velocity $f \dot{z}(t)(f=100 \mathrm{~N} / \mathrm{m} / \mathrm{s})$, a friction proportional to the cart position $k_{s} z(t)\left(k_{s}=0.001 \mathrm{~N} / \mathrm{rad} / \mathrm{s}\right)$ and a friction torque in the angular motion of the pendulum proportional to the angular velocity $k \dot{\theta}(t)(k=0.003 N / \mathrm{rad} / \mathrm{s})$. The state 
equations are as follows:

$$
\left\{\begin{array}{l}
(m+M) \ddot{z}(t)+k_{s} z(t)+f \dot{z}(t)-m l \ddot{\theta}(t) \cos (\theta(t)) \\
+m l \dot{\theta}^{2}(t) \sin (\theta(t))=F(t) \\
-m l \ddot{z}(t) \cos (\theta(t))+\left(m l^{2}+I\right) \ddot{\theta}(t) \\
+k \dot{\theta}(t)+m g l \sin (\theta(t))=0
\end{array}\right.
$$

In order to ensure the nonlinear system stability, the system equations (40) is written under a T-S from by applying the sector nonlinearity approach which allows to exactly represent the nonlinear system without any loss of information. Since the transition from the nonlinear system to the T-S one is not the main subject of this paper and for place limitation, only the main steps are given.

First, the quasi-LPV form (quasi-linear with parameter varying) is derived

$$
\dot{x}(t)=A(x(t)) x(t)+B(x(t)) u(t)
$$

with :

$$
\begin{aligned}
& x(t)=\left(\begin{array}{llll}
z(t) & \dot{z}(t) & \theta(t) & \dot{\theta}(t)
\end{array}\right)^{T}, u(t)=F(t) \\
& A(x(t))=\left(\begin{array}{cccc}
0 & 1 & 0 & 0 \\
-k_{s} /(m+M) & a_{1}(t) & a_{2}(t) & a_{3}(t) \\
0 & 0 & 0 & 1 \\
a_{7}(t) & a_{4}(t) & a_{5}(t) & a_{6}(t)
\end{array}\right) \\
& B(t)=\left(\begin{array}{llll}
0 & b_{1}(t) & 0 & b_{2}(t)
\end{array}\right)^{T}
\end{aligned}
$$

and the parameters $a_{i}(t)$ and $b_{i}(t)$ are defined as follows:

$$
\left\{\begin{array}{l}
a_{1}(t)=-\frac{f}{m+M}-\frac{m l f z_{1}(t) z_{2}^{2}(t)}{m+M} \\
a_{2}(t)=-\frac{m l z_{3}(t) z_{4}(t)}{m+M}- \\
\frac{m^{2} l^{2} z_{2}^{2}(t) z_{1}(t) z_{3}(t) z_{4}(t)}{m+M}-m l g z_{1}(t) z_{2}(t) z_{3}(t) \\
a_{3}(t)=k z_{1}(t) \\
a_{4}(t)=-f z_{1}(t) z_{2}(t) \\
a_{5}(t)=-m l z_{1}(t) z_{2}(t) z_{3}(t) z_{4}(t)-(m+M) g z_{1}(t) z_{3}(t) \\
a_{6}(t)=-\frac{k(m+M)}{m l} z_{1}(t) \\
a_{7}(t)=-k_{s} z_{1}(t) z_{2}(t) \\
b_{1}(t)=\frac{1}{m+M}+\frac{m l}{m+M} z_{2}^{2}(t) z_{1}(t) \\
b_{2}(t)=z_{1}(t) z 2(t)
\end{array}\right.
$$

with the following non-linearity :

$$
\left\{\begin{aligned}
z_{1}(t) & =\frac{1}{-m l \cos ^{2}\left(x_{3}(t)\right)+(l+I / m l)(m+M)} \\
z_{2}(t) & =\cos \left(x_{3}(t)\right) \\
z_{3}(t) & =\frac{\sin \left(x_{3}(t)\right)}{x_{3}} \\
z_{4}(t) & =x_{4}^{2}(t)
\end{aligned}\right.
$$

The reader will note that the nonlinearities choice $\left(z_{1}(t), z_{2}(t), z_{1}(t)\right.$ and $\left.z_{4}(t)\right)$ is not unique and that the premise variables are bounded and given by the angle and speed limitation. Now, the convex polytopic transformation is applied

$$
z_{i}(t)=F_{i 1}(t) z_{i 1}+F_{i 2}(t) z_{i 2}, i=1, \ldots, 4
$$

with

$$
\begin{aligned}
& z_{i 1}=\max z_{i}(t), \quad z_{i 2}=\min z_{i}(t), i=1, \ldots, 4 \\
&\left\{\begin{aligned}
F_{i 1}(t) & =\frac{z_{i}(t)-z_{i 2}}{z_{i 1}-z_{i 2}} \\
F_{i 2}(t) & =\frac{z_{i 1}-z_{i}(t)}{z_{i 1}-z_{i 2}}
\end{aligned}\right.
\end{aligned}
$$

The two partitions $F_{i 1}(t)$ and $F_{i 2}(t)$ of each premise variable $z_{i}(t), i=1,2,3,4$ will contribute to the reconstruction of each weighting function of the T-S model. The sub-model number depends on the non-linearities, such that $r=2^{4}=16$.

The T-S model is then given by the following equations:

$$
\dot{x}(t)=\sum_{i=1}^{16} \mu_{i}(t)\left(A_{i} x(t)+B_{i} u(t)\right)
$$

with

$$
\left\{\begin{array}{l}
\mu_{i}(t)=\prod_{j=1}^{16} F_{j, \sigma_{i}^{j}}\left(z_{j}(t)\right) \\
A_{i}=A\left(z_{1, \sigma_{i}^{1}}, z_{2, \sigma_{i}^{2}}, z_{3, \sigma_{i}^{3}}, z_{4, \sigma_{i}^{4}}\right) \\
B_{i}=B\left(z_{1, \sigma_{i}^{1}}, z_{2, \sigma_{i}^{2}}, z_{3, \sigma_{i}^{3}}, z_{4, \sigma_{i}^{4}}\right)
\end{array}\right.
$$

$\sigma_{i}^{j}$ may take the value 1 or 2 and represents the number of the $j^{\text {th }}$ partition.

In order to simplify the LMI implementation, it is assumed that the control gains $K_{j}$ are equal for all the submodels. Then, two control laws are proposed:

$$
u(t)=-K_{n} x(t) \quad \text { and } \quad u(t)=-K_{T S} x(t)
$$

for the nominal controller (without input saturation) and the proposed T-S controller respectivelly.

The nominal control gain calculated from (21) is equal to:

$$
K_{n}=\left(\begin{array}{llll}
3.4068 & -95.7038 & -29.8001 & 3.8235
\end{array}\right)
$$

Considering the saturation levels equal $u_{\min }=0$ and $u_{\max }=$ 3 , The control gain $K_{T S}$ is then calculated by solving the LMI given by theorem 1 and is equal to:

$$
K_{T S}=\left(\begin{array}{llll}
0.0003 & 5.9835 & 0.6139 & -0.0024
\end{array}\right)
$$

For an initial position $x_{0}=\left(\begin{array}{llll}0 & 0 & \pi / 12 & 0\end{array}\right)^{T}$, figure 2 shows the system states for the following three cases: $x_{n}$ for the nominal case without saturation, $x_{n s a t}$ is the system states with saturated nominal control, it is clear from the depicted figure that the saturation has a destabilizing effect on the system. And finally $x_{\text {satTS }}$ denotes the system state obtained with the proposed T-S approach.

From the depicted figures, it is clear that with the proposed T-S approach the system stability is ensured as well as the convergence to an origin centered-ball. However, since we considered the same gain for all the 16 sub-models, the obtained solution is slightly conservative, which may justify the oscillatory response for the third and fourth state.

In order to relax these constraints, the nonlinear system may be simplified by considering the small angles approximations: $\cos (\theta) \approx 1$ and $\sin (\theta) \approx \theta$. Hence, instead of the four previous non-linearities, only one is considered, namely: $z_{4}(t)=x_{4}^{2}(t)$ and a T-S system with two sub-models is then defined with the T-S controller is then given by

$$
u(t)=-\sum_{i=1}^{2} \mu_{i}(\xi(t)) K_{i T S} x(t)
$$



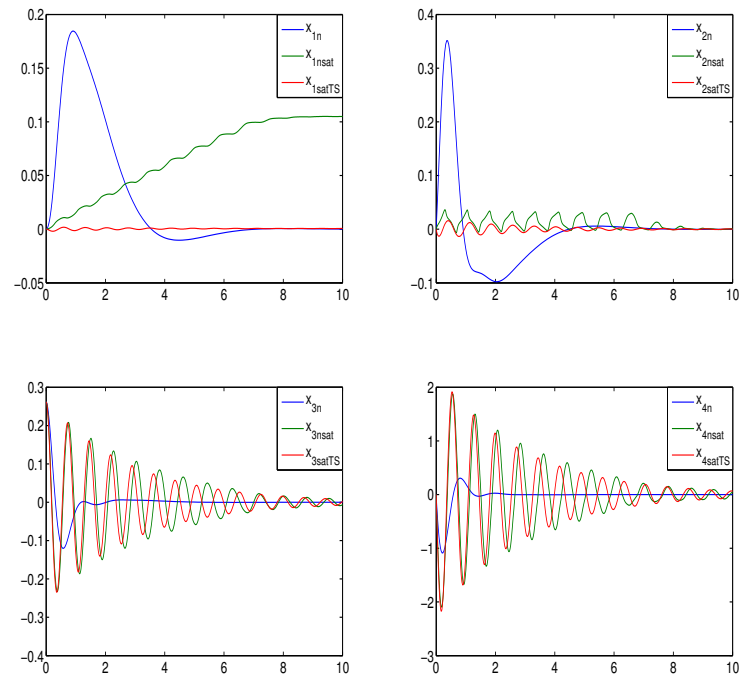

Fig. 2. System states

with

$$
\begin{aligned}
& K_{1 T S}=\left(\begin{array}{llll}
0.0127 & -15.0442 & 15.8757 & 0.7854
\end{array}\right) \\
& K_{2 T S}=\left(\begin{array}{llll}
0.0079 & -19.0341 & 8.7667 & 0.5326
\end{array}\right)
\end{aligned}
$$

As seen on Figure 3, displaying the obtained state responses,
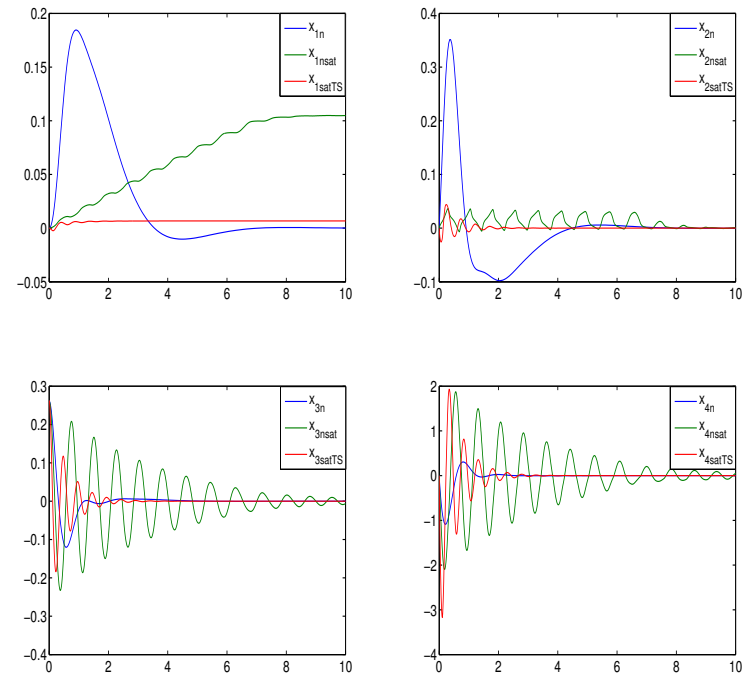

Fig. 3. System states

it is clear that the system states are stable with less oscillations and converge to an origin centered ball.

\section{CONCLUSIONS}

Considering the saturation nonlinearity, a nonlinear system can be represented in an augmented T-S form. It is important to note that, using the proposed representation of the actuator saturation, these saturations are expressed in terms of the control variables and of the saturation limits. As a consequence, these limits are taken into account when solving the LMI optimization problem and when computing the gains of the controller. Moreover, the proposed Takagi-Sugeno approach allows to extend the use of linear tools, namely the LMI formalism, to a nonlinear control problem.

As an illustrative example, the state feedback controller design was applied to a cart-pendulum nonlinear T-S system. The obtained results show the effectiveness of the proposed approach for the studied example.

\section{REFERENCES}

[1] A. Syaichu-Rohman and R. Middleton, "Anti-windup schemes for discrete time systems: An LMI-based design," in $5^{\text {th }}$ Asian Control Conference, Australia, 20-23 July 2004.

[2] L. Zaccarian and A. Teel, "A common framework for anti-windup, bumpless transfer and reliable design," Automatica, vol. 38, no. 10, pp. 1735-1744, 2002.

[3] E. Mulder, M. Kothare, and M. Morari, "Multivariable antiwindup controller synthesis using linear matrix inequalities," Automatica, vol. 37, no. 9 , pp. 1407-1416, 2001.

[4] G. Grimm, J. Hatfield, I. Postlethwaite, A. Teel, M. Turner, and L. Zaccarian, "Antiwindup for stable linear systems with input saturation: an LMI-based synthesis," IEEE Transactions on Automatic Control, vol. 48, no. 9, pp. 1509-1525, 2003.

[5] A. Syaichu-Rohman, "Optimisation based feedback control of input constrained linear systems," Ph.D. dissertation, The University of Newcastle Callaghan, Australia, 2005.

[6] A. Alessandri, M. Baglietto, and G. Battistelli, "On estimation error bounds for receding-horizon filters using quadratic boundedness," IEEE Transactions on Automatic Control, vol. 49, no. 8, pp. 1350-1355, 2004.

[7] F. Blanchini and S. Miani, Set-theoretic methods in control. Systems and Control: Foundations and Applications. Boston-Basel-Berlin: Birkhauser, 2008.

[8] Y. Cao and Z. Lin, "Robust stability analysis and fuzzy-scheduling control for nonlinear systems subject to actuator saturation," IEEE Fuzzy Sets and Systems, vol. 11, no. 1, pp. 57-67, 2003.

[9] S. Tarbouriech, G. Garcia, J. Gomes da Silva Jr., and I. Queinnec, Stability and Stabilization of Linear Systems with Saturating Actuators. London: Springer-Verlag, 2011.

[10] K. Tanaka and H. Wang, Fuzzy Control Systems Design and Analysis: A Linear Matrix Inequality Approach. New York: John Wiley \& Sons, Inc., 2001.

[11] A. Nagy, G. Mourot, B. Marx, J. Ragot, and G. Schutz, "Systematic multimodeling methodology applied to an activated sludge reactor model," Industrial \& Engineering Chemestry Research, vol. 49, no. 6, pp. 2790-2799, 2010.

[12] T. Takagi and M. Sugeno, "Fuzzy identification of systems and its applications to modeling and control," IEEE Transactions on Systems, Man, and Cybernetics, vol. 15, no. 1, pp. 116-132, 1985.

[13] K. Tanaka, T. Hori, and H. Wang, "A multiple Lyapunov function approach to stabilization of fuzzy control systems," IEEE Transactions on Fuzzy Systems, vol. 11, no. 4, pp. 582-589, 2003.

[14] K. Tanaka, H. Ohtake, and H. Wang, "A descriptor system approach to fuzzy control system design via fuzzy Lyapunov functions," IEEE Transactions on Fuzzy Systems, vol. 15, no. 3, pp. 333-341, 2007.

[15] K. Zhang, B. Jiang, and P. Shi, "A new approach to observer-based fault-tolerant controller design for Takagi-Sugeno fuzzy systems with state delay," Circuits, systems, and signal processing, vol. 28, no. 5, pp. 679-697, 2009.

[16] S. Bezzaoucha, B. Marx, D. Maquin, and J. Ragot, "Contribution to the constrained output feedback control," in American Control Conference, ACC, Washington, DC, USA, June 2013. 